\title{
Retraction
}

\section{Retracted: Quality Improvement and Robust Design Methods to a Pharmaceutical Research and Development}

\author{
Mathematical Problems in Engineering \\ Received 16 July 2017; Accepted 16 July 2017; Published 15 November 2017 \\ Copyright (C) 2017 Mathematical Problems in Engineering. This is an open access article distributed under the Creative Commons \\ Attribution License, which permits unrestricted use, distribution, and reproduction in any medium, provided the original work is \\ properly cited.
}

Mathematical Problems in Engineering has retracted the article titled "Quality Improvement and Robust Design Methods to a Pharmaceutical Research and Development" [1]. The article was found to contain a substantial amount of material, without citation, from the following published article: "Cho, B. R., Choi, Y. \& Shin, S. Int J Adv Manuf Technol (2010) 49: 839. doi:10.1007/s00170-009-2455-3” [2]. The authors stated that the previous article used censored data and the current article used noncensored data, which require different techniques to manage. The authors say that in the previous work, type 2 right-censoring schemes were developed and implemented in the robust design context, and thus the basic goals posed and the methods developed in the articles are entirely different. However, much of the text of the articles is the same, including most of the Introduction, Section 2, and Conclusions and parts of Sections 3 and 4.

\section{References}

[1] B. R. Cho and S. Shin, "Quality improvement and robust design methods to a pharmaceutical research and development," Mathematical Problems in Engineering, vol. 2012, Article ID 193246, 2012.

[2] B. R. Cho, Y. Choi, and S. Shin, "Development of censored data-based robust design for pharmaceutical quality by design," International Journal of Advanced Manufacturing Technology, vol. 49, no. 9-12, pp. 839-851, 2010. 


\title{
Quality Improvement and Robust Design Methods to a Pharmaceutical Research and Development
}

\author{
Byung Rae Cho' ${ }^{1}$ and Sangmun Shin ${ }^{2}$ \\ ${ }^{1}$ Department of Industrial Engineering, Clemson University, Clemson, SC 29634, USA \\ ${ }^{2}$ Department of Systems Management \& Engineering, Inje University, \\ Gyeongnam Gimhae 621-749, Republic of Korea
}

Correspondence should be addressed to Sangmun Shin, sshin@inje.ac.kr

Received 19 November 2011; Accepted 18 April 2012

Academic Editor: Dongdong Ge

Copyright (C) 2012 B. R. Cho and S. Shin. This is an open access article distributed under the Creative Commons Attribution License, which permits unrestricted use, distribution, and reproduction in any medium, provided the original work is properly cited.

Researchers often identify robust design, based on the concept of building quality into products or processes, as one of the most important systems engineering design concepts for quality improvement and process optimization. Traditional robust design principles have often been applied to situations in which the quality characteristics of interest are typically time-insensitive. In pharmaceutical manufacturing processes, time-oriented quality characteristics, such as the degradation of a drug, are often of interest. As a result, current robust design models for quality improvement which have been studied in the literature may not be effective in finding robust design solutions. In this paper, we show how the robust design concepts can be applied to the pharmaceutical production research and development by proposing experimental and optimization models which should be able to handle the time-oriented characteristics. This is perhaps the first attempt in the robust design field. An example is given, and comparative studies are discussed for model verification.

\section{Introduction}

Continuous quality improvement has become widely recognized by many industries as a critical concept in maintaining a competitive advantage in the marketplace. It is also recognized that quality improvement activities are efficient and cost-effective when implemented during the design stage. Based on this awareness, Taguchi [1] introduced a systematic method for applying experimental design, which has become known as robust design which is often referred to as robust parameter design. The primary goal of this method is to determine the best design factor settings by minimizing performance variability and product bias, that is, the deviation from the target value of a product. Because of the practicability in reducing 
the inherent uncertainty associated with system performance, the widespread application of robust design techniques has resulted in significant improvements in product quality, manufacturability, and reliability at low cost. Although the main robust design principles have been implemented in a number of different industrial settings, our literature study indicates that robust design has been rarely addressed in the pharmaceutical design process.

In the pharmaceutical industry, the development of a new drug is a lengthy process involving laboratory experiments. When a new drug is discovered, it is important to design an appropriate pharmaceutical dosage or formulation for the drug so that it can be delivered efficiently to the site of action in the body for the optimal therapeutic effect on the intended patient population. The Food and Drug Administration (FDA) requires that an appropriate assay methodology for the active ingredients of the designed formulation be developed and validated before it can be applied to animal or human subjects. Given this fact, one of the main challenges faced by many researchers during the past decades is the optimal design of pharmaceutical formulations to identify better approaches to various unmet clinical needs. Consequently, the pharmaceutical industry's large investment in the research and development $(R \& D)$ of new drugs provides a great opportunity for research in the areas of experimentation and design of pharmaceutical formulations. By definition, pharmaceutical formulation studies are mixture problems. These types of problems take into account the proportions within the mixture, not the amount of the ingredient; thus, the ingredients in such formulations are inherently dependent upon one another, and consequently experimental design methodologies commonly used in many manufacturing settings may not be effective. Instead, for mixture problems, a special kind of experimental design, referred to as a mixture experiment, is needed. In mixture experiments, typical factors in question are the ingredients of a mixture, and the quality characteristic of interest is often based on the proportionality of each of those ingredients. Hence, the quality of the pharmaceutical product is influenced by such designs when they are applied in the early stages of drug development.

In this paper, we propose a new robust design model in the context of pharmaceutical production R\&D. The main contribution of this paper is twofold. First, traditional experimental design methods have often applied to situations in which the quality characteristics of interest are typically time-insensitive. In pharmaceutical manufacturing processes, timeoriented quality characteristics, such as the degradation of a drug, are often of interest, and these time-oriented data often follow a Weibull distribution. Since it may take a long time to observe the degradation of a drug product, the concept of censored samples can be integrated in designing optimal pharmaceutical formulations. In this paper, we develop a censored sample-based experimental design model for optimal pharmaceutical formulations by integrating the main robust design principles. Second, we then show how the response surface methodology, which is a well-established statistical tool, can be integrated with the proposed censored sample-based robust deign model. Finally, we show how the maximum likelihood method is implemented in estimating mean and variance of censored Weibull data. A numerical example is given, and comparison studies for the two estimation methods are discussed for model verification. This paper is organized as follows. In the next section, we present a literature review on mixture design and robust design. In Section 3, we describe our proposed censored robust design model for the optimal design of pharmaceutical formulations in detail. The maximum likelihood method is then studied, and optimization models are proposed. In Section 4, we demonstrate our proposed methods using a numerical example and compare the results under the two different optimization models. In the last section, we conclude the paper with a discussion of our findings. 


\section{Literature Study}

In this section, the literature of robust design and mixture designs is discussed.

\subsection{Robust Design}

Because product performance is directly related to product quality, Taguchi's techniques $[1,2]$ of robust design (RD) have become increasingly popular in industry since the mid 1980s. RD is a powerful and cost-effective quality improvement methodology for products and processes, which results in higher customer satisfaction and operational performance. There is little disagreement among researchers and practitioners about Taguchi's basic philosophy. Steinberg and Bursztyn [3] provided a comprehensive discussion on Taguchi's off-line quality control and showed that the use of noise factors can significantly increase the capability for detecting factors with dispersion effects, when noise factors are explicitly modeled in the analysis. However, the ad hoc robust design methods suggested by Taguchi remain controversial due to various mathematical flaws. The controversy surrounding Taguchi's assumptions, experimental design, and statistical analysis has been well addressed by Leon et al. [4], Box [5], Box et al. [6], Nair [7], and Tsui [8]. Consequently, researchers have closely examined alternative approaches using well-established statistical and optimization tools. Vining and Myers [9] introduced the dual response approach based on response surface methodology (RSM) as an alternative for modeling process relationships by separately estimating the response functions of process mean and variance, thereby achieving the primary goal of robust design by minimizing the process variance while adjusting the process mean at the target. Del Castillo and Montgomery [10] and Copeland and Nelson [11] showed that the optimization technique used by Vining and Myers [9] does not always guarantee optimal robust design solutions and proposed standard nonlinear programming techniques, such as the generalized reduced gradient method and the Nelder-Mead simplex method, which can provide better robust design solutions. The modified dual response approaches using fuzzy theory were further developed by Khattree [12] and Kim and Lin [13]. However, Lin and Tu [14], pointing out that the robust design solutions obtained from the dual response model may not necessarily be optimal since this model forces the process mean to be located at the target value, proposed the mean-squared error model, relaxing the zerobias assumption. While allowing some process bias, the resulting process variance is less than or at most equal to the variance obtained from the Vining and Myers model [9]; hence, the mean-squared error model may provide better (or at least equal) robust design solutions unless the zero-bias assumption must be met. Further modifications to the mean-squared error model have been discussed by Jayaram and Ibrahim [15], Cho et al. [16], Kim and Cho [17, 18], Yue [19], Park and Cho [20], Miro-Quesada and Del Castillo [21], Cho and Park [22], Govindaluri and Cho [23], Shin and Cho [24, 25], and Lee et al. [26]. Along this line, Myers et al. [27], Park and Cho [22], and Robinson et al. [28] developed modified dual-response models using generalized linear model, a robust design model using the weighted-leastsquare method for unbalanced data, and a robust design model using a generalized linear mixed model for nonnormal quality characteristics, respectively. As for an experimental strategy, Kovach and Cho [29-31] and Kovach et al. [32] studied D-optimal robust design problems by minimizing the variance of the regression coefficients. Ginsburg and Ben-Gal [33] then developed a new optimality criterion, called the Vs optimality, which minimizes the variance of the optimal solution by prioritizing the estimation of various model coefficients, thereby, estimating coefficients more accurately at each experimental stage. It is well known 
that estimated empirical models are often subject to random error. In order to obtain a more precise robust design solution in the presence of the error, $\mathrm{Xu}$ and Albin [34] developed a model which can be resistant to the error by considering all points in the confidence intervals associated with the estimated model. When multiple quality characteristics are considered, those characteristics are often correlated. Govindaluri and Cho [23], investigated the effect of correlations of quality characteristics on robust design solutions, while Egorov et al. [35] and Kovach et al. [32] studied optimal robust design solutions using the indirect optimization algorithm and physical programming, respectively. Finally, Shin and Cho [25] studied tradeoff studies on minimizing variance and achieving the predetermined target value.

\subsection{Mixture Designs}

Scheffe [36] first introduced his theory on the prediction of the responses of mixture experiments based on their proportions. The theory defines $x_{i}$ as the proportion of ingredient $i$ in the mixture. Furthermore, the proportionality idea of this theory provides the experiment with a property in which the proportions of the $\mathrm{k}$ ingredients within the mixture must equal 100 percent, as illustrated by the equation $\sum_{i=1}^{k} x_{i}=x_{1}+x_{2}+\cdots+x_{k}=1$, where $x_{i} \geq 0$ for all $i=1,2, \ldots, k$. Scheffe [36] employed a simplex lattice design to represent the design points of the feasible experimental region of the ingredients. The simplex lattice design is defined by the notation $\{k, m\}$, where $m+1$ defines the number of equally spaced proportion values from 0 to 1 for each experiment and those proportions are determined by the equation $x_{i}=0,1 / m, 2 / m, \ldots, 1$. All possible combinations of the proportions are used to determine the design points within the simplex lattice design. In general, the number of design points in a $\{k, m\}$ simplex lattice design is defined $n=(k+m-1) ! / m !(k-1)$ ! Scheffe [37] also modified this simplex lattice design to introduce the simplex centroid design for experiments that include the overall centroid of the region at the coordinate $(1 / k, 1 / k, \ldots, 1 / k)$.

Augmented designs of both the simplex lattice and simplex centroid designs exist. Cornell [38] analyzed both an augmented simplex lattice design and an augmented simplex centroid design with ten design points each. Applications of mixture experiments revealed other design possibilities. The most natural obstacle is the limitation on the proportion of a certain ingredient within a mixture. The limitation could be found in the form of lower, upper, and both lower and upper bounds or constraints. This led researchers to develop other ways to obtain design points that are within the feasible region given the constraints. An example of such models is the extreme vertices design for mixture experiments. First introduced by Mclean and Anderson [39], extreme vertices designs for mixture problems consider the extreme points of the irregular polyhedron, formed by constraints in experimental runs, in addition to the centroids of each facet. The major disadvantage with this design is the possible large number of design points that can be obtained with the given constraints, specifically as the number of ingredients increases and the feasible region becomes more complex. Snee and Marquart [40] presented an algorithm to determine the appropriate subset of design points when the vertices of the feasible region are too many to handle. They compare the efficiency of their approach to G- and D-optimal designs, both of which are common techniques used for determining the appropriate points at which to take observations. Bayesian D-optimal designs shown by DuMouchel and Jones [41] are a modification of D-optimal designs, which reduces the dependency of the design on the assumed model. Using such models as a leverage point, Andere-Rendon et al. [42] investigated the Bayesian D-optimal design specifically for mixture experiments which include both potential and primary model terms 
in order to form the Bayesian slack variable model. The results favored the Bayesian Doptimal design with smaller bias errors and better-fitted models. Along the same lines, Goos and Donev [43] extended the work of Donev [44] with the implementation of D-optimal designs for blocked mixture experiments. Unlike other research that used orthogonally blocked experiments (see $[45,46]$ ), they employed mixture designs that are not orthogonally blocked and used an algorithm that provided a simpler methodology to construct blocked mixture designs.

The simplified polynomials, also referred to as canonical polynomials, are widely used throughout the literature and are embedded in software packages for mixture experiments. However, these designs have been scrutinized, especially because of their lack of squared terms. Piepel et al. [47] proposed a partial quadratic mixture model that includes the linear Scheffé terms but augments them with the appropriate squared or quadratic cross product terms. Extending from alternative models proposed by Snee and Rayner [48], Cornell and Gorman [49] explained how highly constrained regions, such as those in mixture experiments having components with considerably smaller ranges than others, result in skewed responses, thus creating fitted models that have inherent collinearity. Both models attempt to modify the scale on the feasible region that results in the experiment's constraints in an effort to eliminate the collinearity between components. For other collinearity research and discussions, refer to the publications of Sengupta et al. [50] and Prescott et al. [51]. Other research publications have employed robust design methodologies for mixture experiments. Steiner and Hamada [52] modeled a mixture experiment to include the coefficient and terms that account for the interactions of mixture and controllable process variables and the interactions of the mixture and noise variables. This model utilizes the Taguchi loss function $[1,2]$ to reduce the noise variables. The practicality of the design may be questionable as it does not account for constraints within the system, which opens the opportunity for further research. A different approach to dealing with noise factors can be studied in Goldfarb et al. [53]. Continuing in similar research, Goldfarb et al. [54] introduced a three-dimensional variance dispersion graph with the purpose of comparing competing mixture experimental designs based on their prediction variance properties. Goldfarb et al. [55] proposed an interesting addition to this research by implementing genetic algorithms within an objective function to minimize the maximum scaled prediction variance in the mixture design region. This investigation showed that with a few runs of the genetic algorithm, the scaled prediction variance can be significantly reduced, allowing the experimenter to control noise variables inherent in the experiment.

\section{Proposed Censored Robust Design Model}

In this section, we describe the proposed model in three phases-experimental phase, estimation phase, and optimization phase.

\subsection{Notations}

Notations associated with parameters and variables used in paper are defined as follows:

$\mathbf{x}=\left[x_{1}, x_{2}, \ldots, x_{k}\right]$ vector of $k$ control factors,

$\mathbf{y}$ vector of output observations,

$y_{i} i$ output observations,

T censored observations, 
Table 1: General layout of the proposed methodology.

\begin{tabular}{lcccc}
\hline Design points & $\begin{array}{c}\text { Control factors } \\
x_{1}, x_{2}, \ldots, x_{f}\end{array}$ & Experimental observations & \multicolumn{2}{c}{ Estimate } \\
\hline 1 & & $y_{1}, \ldots y_{n_{1}}, T_{1}, \ldots, T_{m_{1}}$ & $\widehat{\mu}_{1}$ & $\widehat{\sigma}_{1}$ \\
2 & & $y_{1}, \ldots y_{n_{2}}, T_{1}, \ldots, T_{m_{2}}$ & $\widehat{\mu}_{2}$ & $\widehat{\sigma}_{2}$ \\
$\vdots$ & Experimental design & $\vdots$ & $\vdots$ & $\vdots$ \\
$i$ & $y_{1}, \ldots y_{n_{i}}, T_{1}, \ldots, T_{m_{i}}$ & $\widehat{\mu}_{i}$ & $\widehat{\sigma}_{i}$ \\
$\vdots$ & $\vdots$ & $\vdots$ & $\vdots$ \\
$d$ & $y_{1}, \ldots y_{n_{d}}, T_{1}, \ldots, T_{m_{d}}$ & $\widehat{\mu}_{d}$ & $\widehat{\sigma}_{d}$ \\
\hline
\end{tabular}

$T_{i} i$ censored observations,

$\boldsymbol{\theta}$ vector of parameter $\theta$,

$\theta_{k}$ the component of vector parameter $\boldsymbol{\theta}$,

$F(\mathbf{y} ; \boldsymbol{\theta})$ cumulative distribution function associated with parameters $y$ and $\boldsymbol{\theta}$,

$f(\mathbf{y} ; \boldsymbol{\theta})$ probability density function associated with parameters $y$ and $\boldsymbol{\theta}$,

$L(\mathbf{y}, \mathbf{T} ; \boldsymbol{\theta})$ maximum likelihood function associated with parameters $y, T$, and $\boldsymbol{\theta}$,

$l(\mathbf{y}, \mathbf{T} ; \boldsymbol{\theta}) \log$ likelihood function of $L(\mathbf{y}, \mathbf{T} ; \boldsymbol{\theta})$,

$\Gamma(\mathbf{x})$ gamma function,

$\widehat{\mu}(\mathbf{x})$ estimated function of process mean,

$\widehat{\sigma}(\mathbf{x})$ estimated function of process variance.

\subsection{Experimental Phase}

Table 1 displays the general framework of the RD methodology, where the $y_{i}$ 's are the observed pharmaceutical quality characteristic values and the $T_{i}$ 's are the censored times. The mean and variance for each design point are estimated using the observations.

\subsection{Estimation Phase}

Observations are of two kinds-actual and censored observations. Assume that the observations follow a distribution with underlying cumulative distribution function $F(\mathbf{y} ; \boldsymbol{\theta})$ and probability density function $f(\mathbf{y} ; \boldsymbol{\theta})$, where $\boldsymbol{\theta}$ is a vector of parameters and $\mathbf{y}$ is a vector of observations. Suppose that for each design point, we have $n$ actual observations $y_{1}, y_{2}, \ldots, y_{n}$ and $m$ censored observations $T_{1}, T_{2}, \ldots, T_{m}$. Then the maximum likelihood function is

$$
L(\mathbf{y}, \mathbf{T} ; \boldsymbol{\theta})=\prod_{i=1}^{n} f\left(y_{i} ; \boldsymbol{\theta}\right) \prod_{j=1}^{m}\left[1-F\left(T_{j} ; \boldsymbol{\theta}\right)\right]
$$


and the loglikelihood function is

$$
l(\mathbf{y}, \mathbf{T} ; \boldsymbol{\theta})=\sum_{i=1}^{n} \ln f\left(y_{i} ; \boldsymbol{\theta}\right)+\sum_{j=1}^{m} \ln \left[1-F\left(T_{j} ; \boldsymbol{\theta}\right)\right] .
$$

If the censoring time is fixed at $T$, say, then $\prod_{j=1}^{m}\left[1-F\left(T_{j} ; \boldsymbol{\theta}\right)\right]=[1-F(T ; \boldsymbol{\theta})]^{m}$. In that case, the loglikelihood function is

$$
l(\mathbf{y}, \mathbf{T} ; \boldsymbol{\theta})=\sum_{i=1}^{n} \ln f\left(y_{i} ; \boldsymbol{\theta}\right)+m \ln [1-F(T ; \boldsymbol{\theta})]
$$

The values of the components of $\boldsymbol{\theta}$ that maximize the loglikelihood function will constitute the maximum likelihood estimates. These are computed as the solutions to the system of equations:

$$
\frac{\partial}{\partial \theta_{k}} l(\mathbf{y}, \mathbf{T} ; \boldsymbol{\theta})=0
$$

where the $\theta_{k}$ 's are the components of the vector of parameters $\boldsymbol{\theta}$. If the underlying distribution follows a Weibull distribution with parameters $\alpha$ and $\beta$, then $\boldsymbol{\theta}=[\alpha, \beta]$, and for the $n$ actual observations $y_{1}, y_{2}, \ldots, y_{n}$, and $m$ censored observations $T_{1}, T_{2}, \ldots, T_{m}$, the loglikelihood function is

$$
l(\mathbf{y}, \mathbf{T} ; \mu ; \sigma)=n(\ln \alpha+\ln \beta)+\sum_{i=1}^{n}(\beta-1) \ln y_{i}-\alpha \sum_{i=1}^{n} y_{i}^{\beta}-\alpha \sum_{j=1}^{m} T_{j}^{\beta} .
$$

For a fixed censoring time $T$,

$$
l(\mathbf{y}, \mathbf{T} ; \mu ; \sigma)=n(\ln \alpha+\ln \beta)+\sum_{i=1}^{n}(\beta-1) \ln y_{i}-\alpha \sum_{i=1}^{n} y_{i}^{\beta}-\alpha m T .
$$

The maximum likelihood estimates of $\alpha$ and $\beta$ are the solutions to the system of equations:

$$
\begin{gathered}
\frac{n}{\alpha}-\sum_{i=1}^{n} y_{i}^{\beta}-m T^{\beta}=0 \\
\frac{n}{\beta}+\sum_{i=1}^{n} \ln y_{i}-\alpha \sum_{i=1}^{n} y_{i}^{\beta} \ln y_{i}-\alpha m T^{\beta} \ln T=0
\end{gathered}
$$

Equation (3.7) is obtained by taking the derivatives of the loglikelihood function with respect to $\alpha$ and $\beta$. Similarly, a system of equations can be derived using (3.6) for varying censoring 
times. The solutions to (3.7), namely, $\widehat{\alpha}$ and $\widehat{\beta}$, are the maximum likelihood estimates of $\alpha$ and $\beta$, and we use them in estimating the mean and standard deviation as follows:

$$
\begin{gathered}
\widehat{\mu}=\widehat{\alpha}^{1 / \widehat{\beta}} \Gamma\left(1+\frac{1}{\widehat{\beta}}\right), \\
\widehat{\alpha}=\sqrt{\widehat{\alpha}^{2 / \widehat{\beta}} \Gamma\left(1+\frac{2}{\widehat{\beta}}\right)-\widehat{\mu}^{2} .}
\end{gathered}
$$

\subsection{Optimization Phase}

The main objective of the proposed robust design is to obtain the optimal pharmaceutical formulation settings by maximizing mean response while minimizing variability. Thus, in order to meet this goal, we seek to maximize the mean response while minimizing the variability. To achieve this objective, we propose the following optimization model (Model 1):

$$
\begin{array}{ll}
\min _{\mathbf{x} \in \Omega} & \left\{-\mu^{2}(\mathbf{x})+\sigma^{2}(\mathbf{x})\right\} \\
\text { s.t. } & \sum_{i=1}^{k} x_{k}=1 .
\end{array}
$$

It is noted that the sum of pharmaceutical component proportion is one. By considering the usual approximation of the Taguchi's loss function [1], we can also find the solution to the following optimization model (Model 2):

$$
\begin{aligned}
& \min _{\mathbf{x} \in \Omega}\left\{\frac{1}{\mu^{2}(\mathbf{x})}\left(1+3 \frac{\sigma^{2}(\mathbf{x})}{\mu^{2}(\mathbf{x})}\right)\right\} \\
& \text { s.t. } \quad \sum_{i=1}^{k} x_{k}=1 .
\end{aligned}
$$

By inspection, we notice that this function decreases as $\mu(\mathbf{x})$ increases and as $\sigma(\mathbf{x})$ decreases. Also, a part of the feasibility requirements for these proposed objective functions is that the mean response is nonzero, that is, $\mu(\mathbf{x}) \neq 0$, which is the case for censored samples where the objective is to get $\mu(\mathbf{x})$ as large as possible. We will demonstrate that both proposed optimization models yield optimal solutions in the next section.

\section{Numerical Example and Comparison Study}

Consider an experiment on the degradation of a drug where the factors of concern are corn starch $\left(x_{1}\right)$, saccharin sodium $\left(x_{2}\right)$, and dextrose $\left(x_{3}\right)$. Thus, the vector of the control factors is $\mathbf{x}=\left[x_{1}, x_{2}, x_{3}\right]$. The objective of the experiment is to determine the settings of the control factors, $\mathbf{x}^{*}=\left[x_{1}^{*}, x_{2}^{*}, x_{3}^{*}\right]$, that give the longest possible degradation and the smallest possible variability. The chosen design is a mixture design for three control factors. Suppose 
Table 2: Mixture experiments and data.

\begin{tabular}{lccl}
\hline$x_{1}$ & $x_{2}$ & $x_{3}$ & Observations \\
\hline 1 & 0 & 0 & $\mathbf{3 0 +}, \mathbf{3 0 +}, 27.3130,30+, 25.3841,18.4713,26.3209,25.1345,32.6499, \mathbf{3 0 +}$ \\
0 & 1 & 0 & $\mathbf{3 0 +}, 20.5247,9.9338,22.1908,24.0196,16.9328,18.3113,21.5325,21.4749,17.7806$ \\
0 & 0 & 1 & $11.6089,24.6178,18.2190,21.9588,27.6887,21.6017,26.7790,13.8795,24.6055,30+$ \\
$1 / 2$ & $1 / 2$ & 0 & $24.8139,27.3573,30+, 23.8765,30+, 15.8716,30+, 30+, 25.4216,30+$ \\
$1 / 2$ & 0 & $1 / 2$ & $29.3682,30+, 34.0853,28.2447,21.2239,19.7356,30+, 23.1654,20.4046$ \\
0 & $1 / 2$ & $1 / 2$ & $23.8148,14.5317,25.6167,23.4480,30+, 18.7073,30+, 41.6825,24.0814,14.2608$ \\
$1 / 6$ & $1 / 6$ & $2 / 3$ & $30+, 27.6718,30+, 27.1105,18.4573,28.0881,30+, 28.7328,21.7883,23.6375$ \\
$1 / 6$ & $2 / 3$ & $1 / 6$ & $14.8984,19.5179,27.8163,24.6057,30+, 26.3950,28.0956,18.4866,18.7682,27.7717$ \\
$2 / 3$ & $1 / 6$ & $1 / 6$ & $23.0132,25.5782,30+, 30+, 28.1479,30+, 30+, 30+, 19.7557,30+$ \\
$2 / 3$ & $1 / 3$ & $1 / 3$ & $30+, 28.9475,19.3873,30+, 27.4342,28.5594,30+, 30+, 17.7312,21.8007$ \\
\hline
\end{tabular}

Table 3: Parameter estimates.

\begin{tabular}{lccccc}
\hline Design point & $x_{1}$ & $x_{2}$ & $x_{3}$ & \multicolumn{2}{c}{ Estimate } \\
\hline 1 & 1 & 0 & 0 & 25.6885 & 0.5176 \\
2 & 0 & 1 & 0 & 18.2861 & 5.0843 \\
3 & 0 & 0 & 1 & 20.8556 & 5.5625 \\
4 & $1 / 2$ & $1 / 2$ & 0 & 22.3393 & 5.4712 \\
5 & $1 / 2$ & 0 & $1 / 2$ & 25.1136 & 5.4931 \\
6 & 0 & $1 / 2$ & $1 / 2$ & 23.3288 & 8.4396 \\
7 & $1 / 6$ & $1 / 6$ & $2 / 3$ & 24.5420 & 4.1651 \\
8 & $1 / 6$ & $2 / 3$ & $1 / 6$ & 22.6876 & 4.9773 \\
9 & $2 / 3$ & $1 / 6$ & $1 / 6$ & 22.8861 & 5.1334 \\
10 & $2 / 3$ & $1 / 3$ & $1 / 3$ & 23.3731 & 5.3724 \\
\hline
\end{tabular}

10 samples are subject to each design point, and the experiment is run for 30 hours. The manufacturer believes that the degradation times follow a Weibull distribution. Notice that this is a censoring problem because the experiment is terminated after 30 hours, and for the rest of the samples denoted by 30+, we only know that their degradation times exceed 30 hours but we do not know their actual times. Using the functions in the methods of Sections 3.2 and 3.3, estimates of the means and standard deviations are obtained for each design point. Table 2 shows the experimental design together with the observed responses that have failed. Note that the number of observations per design point is not the same throughout the experiment.

In Table 3, we show the estimates of the mean and standard deviation obtained by our proposed algorithm and by the method of ML estimation. Using the MLE algorithm, the response surfaces for the mean and standard deviation are, respectively, found as

$$
\begin{aligned}
& \widehat{\mu}(\mathbf{x})=27.222 x_{1}+18.601 x_{2}+2.190 x_{3}-0.318 x_{1} x_{2}+5.680 x 1_{x} 3+14.908 x_{2} x_{3}, \\
& \widehat{\sigma}(\mathbf{x})=4.884 x_{1}+5.088 x_{2}+5.300 x_{3}-0.568 x_{1} x_{2}-1.917 x 1_{x} 3+7.957 x_{2} x_{3} .
\end{aligned}
$$


Response surface plots of the process mean
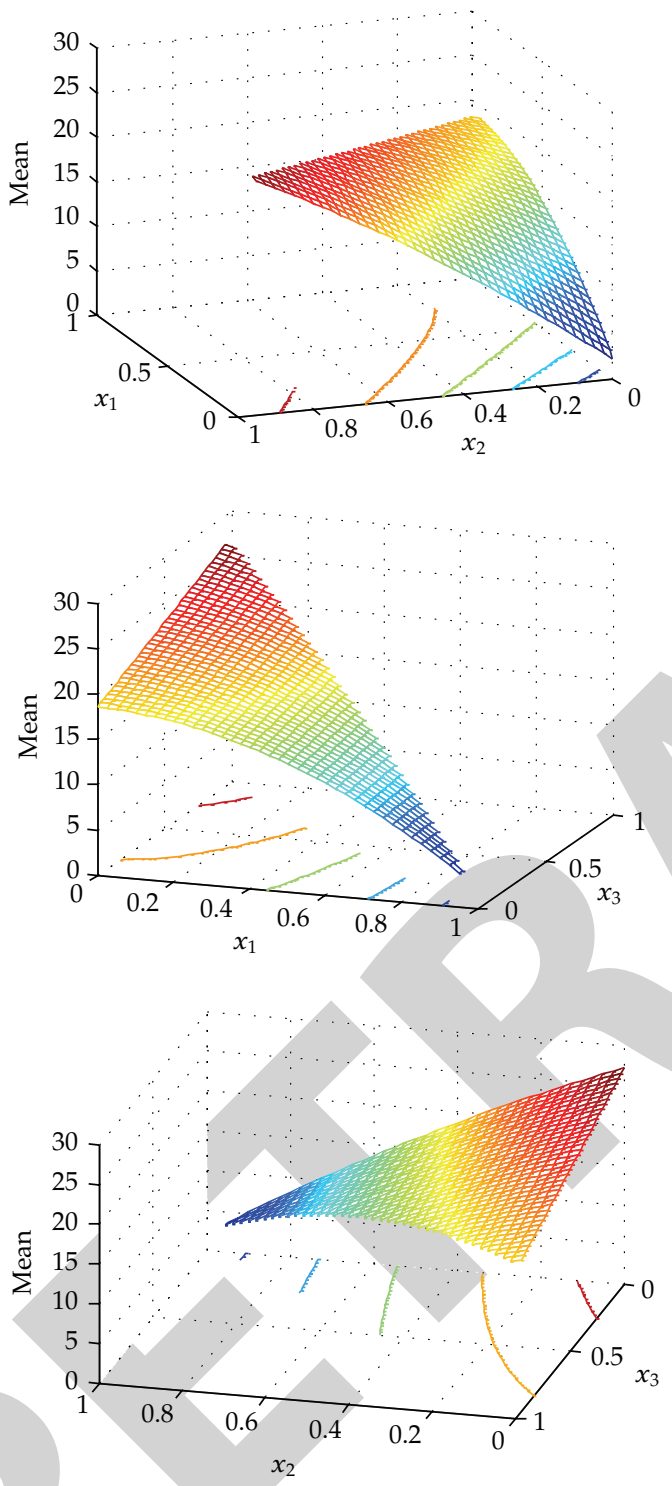

Response surface plots of the process standard deviation

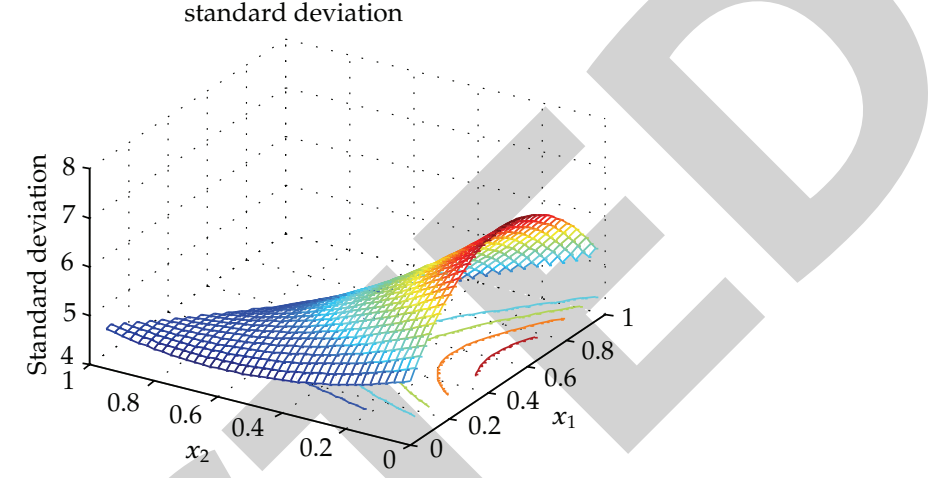

Figure 1: Response surface plots of the mean and standard deviation.

Using (4.1), Figure 1 illustrates surface plots of the process mean and standard deviation associated with input control factors $\left(x_{1}, x_{2}, x_{3}\right)$. In order to represent tradeoffs between the mean and standard deviation, a criterion space is demonstrated in Figure 2.

Table 4 shows the results obtained using the two different optimization models we studied earlier. For this particular example, Model 1 is more effective in increasing the mean value, while Model 2 turns out be to more effective in decreasing standard deviation. The basic goal of both optimization models is to obtain the optimal factor level settings which maximize the mean and minimize the standard deviation. We recommend practitioners use 


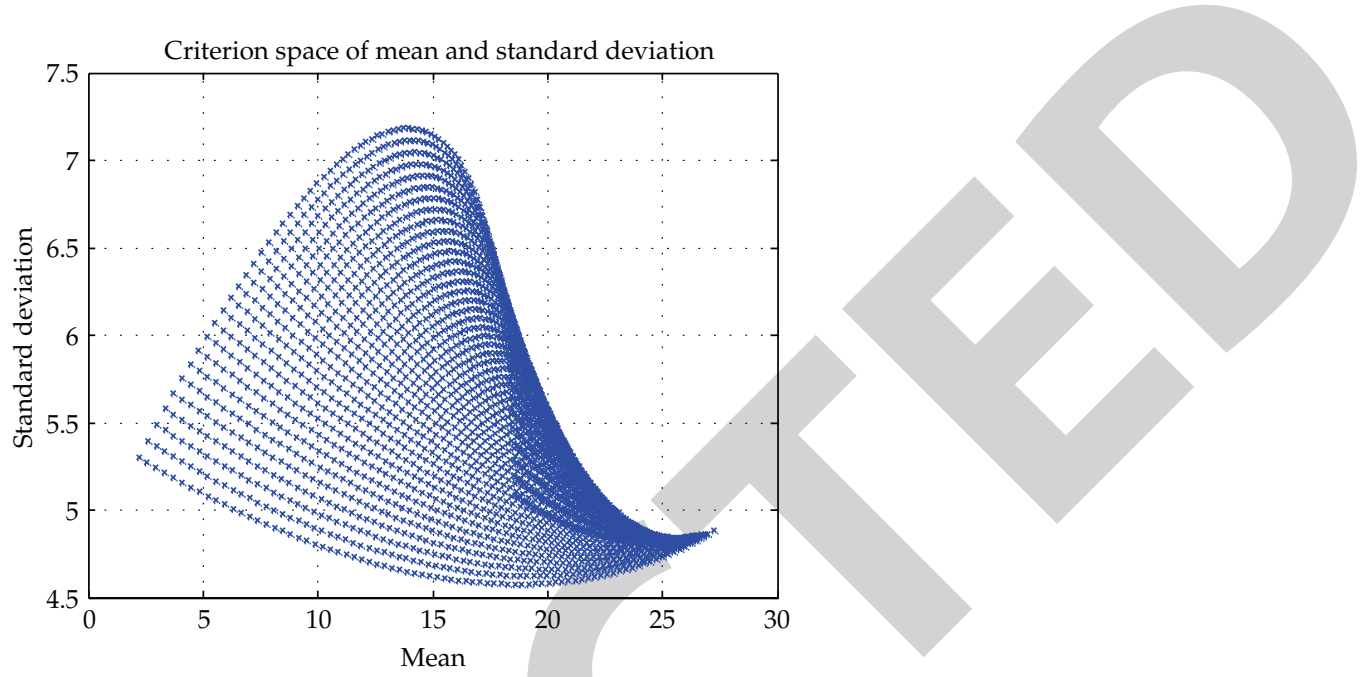

Figure 2: Criterion space for the mean and standard deviation.

Table 4: Optimal settings under two models.

\begin{tabular}{lccc}
\hline Design & Opt settings & Opt mean & Opt Std Dev \\
& $x^{*}=\left[x_{1}^{*}, x_{2}^{*}, x_{3}^{*}\right]$ & $\widehat{\mu}\left(x^{*}\right)$ & $\widehat{\sigma}\left(x^{*}\right)$ \\
\hline Model 1 based on (11) & {$[0.0000,0.6587,0.3413]$} & 27.1974 & 6.9492 \\
Model 2 based on (12) & {$[0.3332,0.3336,0.3333]$} & 26.1309 & 5.6933 \\
\hline
\end{tabular}

both optimization models and pick one of them, depending on their priority of mean and standard deviation.

\section{Conclusions}

Robust design has been demonstrated to be a successful process improvement methodology for diverse system engineering problems. However, its ability to provide sound solutions for time-oriented data is currently limited since the traditional robust design method is mainly applicable to time-insensitive data, and an appropriate robust design method for time-oriented data has not been reported. This paper has proposed new robust design experimental and optimization models for time-oriented data by developing a set of methods in three separate phases, namely, modeling, estimation, and optimization phases. More specifically, this paper has proposed a censored sample-based robust design model by developing a censored experimental design framework, a censored maximum likelihood estimation method in the context of robust design, and optimization models for the censored robust design. For verification, a proposed optimization model has been compared with the traditional Taguchi optimization model. The proposed experimental methodology is particularly useful for experiments with time-oriented pharmaceutical characteristics, such as the degradation of a drug, with an unequal number of observations at each design point. With suitable modifications, our proposed methodology could be extended to the case in which multiple quality characteristics are often correlated under study. Another area for 
future study includes the development of optimal designs, known as computerized designs, for the case in which physical experimental constraints are imposed.

\section{Acknowledgment}

This work was supported by the 2011 Inje University research grant.

\section{References}

[1] G. Taguchi, Introduction to Quality Engineering, Asian Productivity Organization, UNIPUB/Kraus International, White Plains, NY, USA, 1986.

[2] G. Taguchi, Systems of Experimental Design: Engineering Methods to Optimize Quality and Minimize Cost, UNIPBUK/Kraus International, White Plains, NY, USA, 1987.

[3] D. M. Steinberg and D. Bursztyn, "Noise factors, dispersion effects, and robust design," Statistica Sinica, vol. 8, no. 1, pp. 67-85, 1998.

[4] R. V. León, A. C. Shoemaker, and R. N. Kacker, "Performance measures independent of adjustment. An explanation and extension of Taguchi's signal-to-noise ratios," Technometrics, vol. 29, no. 3, pp. 253-285, 1987.

[5] G. E. P. Box, “Signal-to-noise ratios, performance criteria, and transformations," Technometrics, vol. 30, no. 1, pp. 1-17, 1988.

[6] G. E. P. Box, S. Bisgaard, and C. Fung, "An explanation and critique of Taguchi's contributions to quality engineering," Quality and Reliability Engineering International, vol. 4, no. 2, pp. 123-131, 1988.

[7] V. N. Nair, "Taguchi's parameter design: a panel discussion," Technometrics, vol. 34, no. 2, pp. 127-161, 1992.

[8] K. L. Tsui, "An overview of Taguchi method and newly developed statistical methods for robust design," IIE Transactions, vol. 24, pp. 44-57, 1992.

[9] G. G. Vining and R. H. Myers, "Combining Taguchi and response surface philosophies: a dual response approach," Journal of Quality Technology, vol. 22, pp. 38-45, 1990.

[10] E. Del Castillo and D. C. Montgomery, "A nonlinear programming solution to the dual response problem," Journal of Quality Technology, vol. 25, no. 3, pp. 199-204, 1993.

[11] K. A. F. Copeland and P. R. Nelson, "Dual response optimization via direct function minimization," Journal of Quality Technology, vol. 28, no. 3, pp. 331-336, 1996.

[12] R. Khattree, "Robust parameter design: a response surface approach," Journal of Quality Technology, vol. 28, no. 2, pp. 187-198, 1996.

[13] K. J. Kim and D. K. J. Lin, "Dual response surface optimization: a fuzzy modeling approach," Journal of Quality Technology, vol. 30, no. 1, pp. 1-10, 1998.

[14] D. K. J. Lin and W. Tu, “Dual response surface optimization," Journal of Quality Technology, vol. 27, no. 1, pp. 34-39, 1995.

[15] J. S. R. Jayaram and Y. Ibrahim, “Multiple response robust design and yield maximization," International Journal of Quality and Reliability Management, vol. 6, pp. 826-837, 1999.

[16] B. R. Cho, Y. J. Kim, D. L. Kimbler, and M. D. Phillips, "An integrated joint optimization procedure for robust and tolerance design," International Journal of Production Research, vol. 38, no. 10, pp. 2309-2325, 2000.

[17] Y. J. Kim and B. R. Cho, "Economic integration of design optimization," Quality Engineering, vol. 12, no. 4, pp. 561-567, 2000.

[18] Y. J. Kim and B. R. Cho, "Determining the optimum process mean for a skewed process," International Journal of Industrial Engineering, vol. 10, no. 4, pp. 555-561, 2003.

[19] R.-X. Yue, "Model-robust designs in multiresponse situations," Statistics \& Probability Letters, vol. 58, no. 4, pp. 369-379, 2002.

[20] C. Park and B. R. Cho, "Development of robust design under contaminated and non-normal data," Quality Engineering, vol. 15, no. 3, pp. 463-469, 2003.

[21] G. Miro-Quesada and E. Del Castillo, "A dual response approach to the multivariate robust parameter design problem," Technometrics, vol. 46, no. 2, pp. 176-187, 2004.

[22] B. R. Cho and C. Park, "Robust design modeling and optimization with unbalanced data," Computers and Industrial Engineering, vol. 48, no. 2, pp. 173-180, 2005. 
[23] S. M. Govindaluri and B. R. Cho, "Integration of customer and designer preferences in robust design," International Journal of Six Sigma and Competitive Advantage, vol. 1, no. 3, pp. 276-294, 2005.

[24] S. Shin and B. R. Cho, "Bias-specified robust design optimization and its analytical solutions," Computers and Industrial Engineering, vol. 48, no. 1, pp. 129-140, 2005.

[25] S. Shin and B. R. Cho, "Studies on a biobjective robust design optimization problem," IIE Transactions, vol. 41, no. 11, pp. 957-968, 2009.

[26] S. B. Lee, C. Park, and B. R. Cho, “Development of a highly efficient and resistant robust design," International Journal of Production Research, vol. 45, no. 1, pp. 157-167, 2007.

[27] W. R. Myers, W. A. Brenneman, and R. H. Myers, "A dual-response approach to robust parameter design for a generalized linear model," Journal of Quality Technology, vol. 37, no. 2, pp. 130-138, 2005.

[28] T. J. Robinson, S. S. Wulff, D. C. Montgomery, and A. I. Khuri, "Robust parameter design using generalized linear mixed models," Journal of Quality Technology, vol. 38, no. 1, pp. 65-75, 2006.

[29] J. Kovach and B. R. Cho, "A D-optimal design approach to robust design under constraints: a new Design for Six Sigma tool," International Journal of Six Sigma and Competitive Advantage, vol. 2, no. 4, pp. 389-403, 2006.

[30] J. Kovach and B. R. Cho, "Development of a D-optimal robust design model for restricted experiments," International Journal of Industrial Engineering, vol. 14, pp. 117-128, 2007.

[31] J. Kovach and B. R. Cho, "Solving multiresponse optimization problems using quality function-based robust design," Quality Engineering, vol. 20, no. 3, pp. 346-360, 2008.

[32] J. Kovach, B. R. Cho, and J. Antony, "Development of an experiment-based robust design paradigm for multiple quality characteristics using physical programming," International Journal of Advanced Manufacturing Technology, vol. 35, no. 11-12, pp. 1100-1112, 2008.

[33] H. Ginsburg and I. Ben-Gal, "Designing experiments for robust-optimization problems: the Vs-optimality criterion," IIE Transactions, vol. 38, no. 6, pp. 445-461, 2006.

[34] D. Xu and S. L. Albin, "Robust optimization of experimentally derived objective functions," IIE Transactions, vol. 35, no. 9, pp. 793-802, 2003.

[35] I. N. Egorov, G. V. Kretinin, I. A. Leshchenko, and S. V. Kuptzov, “Multi-objective approach for robust design optimization problems," Inverse Problems in Science and Engineering, vol. 15, no. 1, pp. 47-59, 2007.

[36] H. Scheffé, "Experiments with mixtures," Journal of the Royal Statistical Society. Series B, vol. 20, pp. 344-360, 1958.

[37] H. Scheffé, "The simplex-centroid design for experiments with mixtures," Journal of the Royal Statistical Society. Series B, vol. 25, pp. 235-263, 1963.

[38] J. A. Cornell, Experiments with Mixtures: Designs, Models, and the Analysis of Mixture Data, John Wiley \& Sons Inc., New York, NY, USA, 1981.

[39] R. A. McLean and V. L. Anderson, "Linear estimates of parameters in the extreme value distribution," Technometrics, vol. 8, pp. 454-477, 1966.

[40] D. D. Snee and D. W. Marquardt, "Extreme vertices designs for linear mixture models," Technometrics, vol. 16, no. 3, pp. 399-408, 1974.

[41] W. DuMouchel and B. Jones, "A simple bayesian modification of d-optimal designs to reduce dependence on an assumed model," Technometrics, vol. 36, pp. 37-47, 1994.

[42] J. Andere-Rendon, D. C. Montgomery, and D. A. Rollier, "Design of mixture experiments using bayesian D-optimality," Journal of Quality Technology, vol. 29, no. 4, pp. 451-463, 1997.

[43] P. Goos and A. N. Donev, "The D-optimal design of blocked experiments with mixture components," Journal of Quality Technology, vol. 38, no. 4, pp. 319-332, 2006.

[44] A. N. Donev, "Design of experiments with both mixture and qualitative factors," Journal of the Royal Statistical Society. Series B, vol. 51, no. 2, pp. 297-302, 1989.

[45] A. K. Nigam, "Correction to: "Block designs for mixture experiments", " The Annals of Statistics,vol. 4, no. 6, pp. 1294-1295, 1976.

[46] P. W. M. John, “Experiments with mixtures involving process variables," Tech. Rep. 8, Center for Statistical Sciences, University of Texas, Austin, Tex, USA, 1984.

[47] G. F. Piepel, J. M. Szychowski, and J. L. Loeppky, “Augmenting Scheffé linear mixture models with squared and/or crossproduct terms," Journal of Quality Technology, vol. 34, no. 3, pp. 297-314, 2002.

[48] R. D. Snee and A. A. Rayner, "Assessing the accuracy of mixture model regression calculations," Journal of Quality Technology, vol. 14, no. 2, pp. 67-79, 1982. 
[49] J. A. Cornell and J. W. Gorman, "Fractional design plans for process variables in mixture experiments," Journal of Quality Technology, vol. 16, no. 1, pp. 20-38, 1984.

[50] T. K. Sengupta, R. K. Nandy, S. Mukhopadhyay et al., “Mixture models based on homogeneous polynomials," Journal of Statistical Planning and Inference, vol. 71, no. 1-2, pp. 303-311, 1998.

[51] P. Prescott, N. R. Draper, A. M. Dean, and S. M. Lewis, "Mixture experiments: ILL-conditioning and quadratic model specification," Technometrics, vol. 44, no. 3, pp. 260-268, 2002.

[52] S. H. Steiner and M. Hamada, "Making mixtures robust to noise and mixing measurement errors," Journal of Quality Technology, vol. 29, no. 4, pp. 441-450, 1997.

[53] H. B. Goldfarb, C. M. Borror, and D. C. Montgomery, "Mixture-process variable experiments with noise variables," Journal of Quality Technology, vol. 35, no. 4, pp. 393-405, 2003.

[54] H. B. Goldfarb, C. M. Borror, D. C. Montgomery, and C. M. Anderson-Cook, "Three-dimensional variance dispersion graphs for mixture-process experiments," Journal of Quality Technology, vol. 36, no. 1, pp. 109-124, 2004.

[55] H. B. Goldfarb, C. M. Borror, D. C. Montgomery, and C. M. Anderson-Cook, "Using genetic algorithms to generate mixture-process experimental designs involving control and noise variables," Journal of Quality Technology, vol. 37, no. 1, pp. 60-74, 2005. 